\title{
Apoptosis: Reprogramming and the Fate of Mature Cells
}

\author{
Hoi-Hung Cheung, Xiaozhuo Liu, and Owen M. Rennert \\ Section on Clinical and Developmental Genomics, The Eunice Kennedy Shriver National Institute of Child Health \\ and Human Development, National Institutes of Health, Bethesda, MD 20892, USA
}

Correspondence should be addressed to Owen M. Rennert, rennerto@mail.nih.gov

Received 9 January 2012; Accepted 14 February 2012

Academic Editors: D. Arnoult and R. Poon

Copyright ( $\odot 2012$ Hoi-Hung Cheung et al. This is an open access article distributed under the Creative Commons Attribution License, which permits unrestricted use, distribution, and reproduction in any medium, provided the original work is properly cited.

\begin{abstract}
Apoptosis is essential for embryogenesis, organ metamorphosis, and tissue homeostasis. In embryonic stem cells, self-renewal is balanced with proliferative potential, inhibition of differentiation, and prevention of senescence and apoptosis. Growing evidence supports the role of apoptosis in self-renewal, differentiation of pluripotent stem cells, and dedifferentiation (reprogramming) of somatic cells. In this paper we discuss the multiple roles of apoptosis in embryonic stem cells (ESCs) and reprogramming of differentiated cells to pluripotency. The role of caspases and p53 as key effectors in controlling the generation of iPSC is emphasized. Remarkably, the complication of apoptosis arising during reprogramming may provide insights into technical improvements for derivation of iPSC from senescent cells as a tool for modeling aging-related diseases.
\end{abstract}

\section{Introduction}

Apoptosis is a type of programmed cell death first described by Kerr et al. in the 1970s. It is morphologically characterized by cell shrinkage, membrane blebbing and nuclear condensation, and formation of apoptotic bodies [1]. The apoptotic process is initiated by "death" signals, which trigger a complex series of events with multiple positive and negative feedback loops [2]. A central step in the execution of the apoptotic process is the activation of caspases, a group of enzymes belonging to the cysteine protease family. Activation of caspases cleaves many vital cellular proteins, breaking down the nuclear scaffold and cytoskeleton, and subsequently leads to nuclear DNA degradation [3].

Based on the original source of "death" signals, caspasedependent apoptosis is classified into two pathways: intrinsic pathway, which is activated by modulators within the cell itself, and extrinsic pathway, which responds mainly to extracellular stimuli [4]. In the extrinsic pathway (also known as receptor-mediated death pathway), death ligands such as TRAIL and FasL induce apoptosis by activating the death receptors (DR4/TRAILR1/Apo2 and Fas/Apo1, etc.) at the cell surface. The activated death receptors oligomerize, which leads to recruitment of the adaptor proteins such as FADD
(Fas-associated protein with death domain) and further chain activation of caspase- 8 and $-3[2,4,5]$. The intrinsic pathway, which is also known as mitochondria-mediated death pathway, is initiated by damage to mitochondria which results in the release of a series of proteins into cytoplasm, including cytochrome $\mathrm{c}$. When released into the cytoplasm, cytochrome c complexes with Apaf-1, and this complex further activates caspase- 9 and ultimately caspase-3. Activated caspase-3 further cleaves critical intracellular proteins and thereby induces the final stage of cell death [4-6].

A critical event for the intrinsic pathway is release of cytochrome $\mathrm{c}$ from mitochondria, a step strictly regulated by members of the B-cell lymphoma $2(\mathrm{Bcl}-2)$ family $[6,7]$. Members of this family are divided into two subfamilies: one includes the proapoptotic proteins such as Bak, Bax, and Bid; another includes the antiapoptotic proteins such as $\mathrm{Bcl}-2$ and Bcl-xL [5]. Recent studies suggest that induction of pro-apoptotic proteins and inhibition of anti-apoptotic proteins, triggered by certain anti-cancer agents, directly alter the permeability of mitochondrial membrane and further induce the apoptosis signaling pathway by release of cytochrome $\mathrm{c}$ and/or SMAC/Diablo [5,8].

Apart from the Bcl-2 family, p53 protein, a well-known tumor suppressor protein, is also a key player in apoptosis. 
p53 can "sense" DNA damage and trigger a transient cell cycle arrest to allow DNA repair. In the situation of extensive DNA damage, p53 will promote irreversible cell cycle arrest (senescence) or apoptosis [9]. Therefore, p53 is considered as a key guardian of the genome [10]. p53 can induce both the intrinsic and extrinsic apoptotic pathways [11]. It has been reported to be involved in the caspase-8-dependent intrinsic pathway [12]. In addition to direct activation of Bax, p53 can trigger caspase-8-dependent Bid cleavage, which results in Bax conformation and induces cytochrome $\mathrm{c}$ release into the cytosol from mitochondria [13].

Collectively, apoptosis is essential for the sophisticated architecture of life including normal cell turnover, organ metamorphosis, tissue homeostasis, and embryonic development $[7,14-17]$. Here we briefly introduce the general background of apoptosis. The details of the molecular players participating in apoptosis have previously been reviewed $[3,18,19]$.

In this paper we briefly summarize some recent studies on apoptotic events found in embryonic stem cells (ESCs), and link them to the cellular reprogramming, with the emphasis on the role of p53 and caspases, that reverses differentiated cells to an undifferentiated state. We also discuss the crosstalk between reprogramming, accelerated aging, and senescence.

\section{Apoptosis in Human Embryonic Stem Cells}

Human embryonic stem cells (hESCs) have evoked more and more interest from biomedical and developmental researchers since the first hESC line was derived in 1998, as these pluripotent cells provide an opportunity to develop essential treatments for currently incurable diseases; in addition cultured hESCs offer a useful in vitro system to study the processes of human development [20-22].

hESCs, the pluripotent cell population derived from the blastocyst, are characterized by unlimited self-renewal and pluripotency, the ability to differentiate into all cell types representing all three germ layers both in vitro and in vivo. Stem cells are at higher risk of accumulating potentially deleterious mutations than differentiated somatic cells since stem cells have a higher potential for self-renewal $[7,23]$. Inadequate genomic stability is particularly deleterious for hESCs capacity for self-renewal and organismic development, since they are the progenitors of all organ systems, including germ cells [23]. Therefore, hESCs have a more stringent mechanism to ensure their genomic stability and integrity than differentiated cells. There is some evidence to support this postulation, including the following: (1) lower mutation frequency (such as errors in replication, point mutation) is observed in ESCs than in somatic cells; (2) abnormal ESCs with DNA damage are removed from the self-renewal pluripotent population through apoptotic or differentiation programs $[23,24]$. As a guardian of the genome, p53 has been shown to be a critical component, but its role in the induction of apoptosis is independent of its classic action in nuclear accumulation and induction of cell cycle arrest [25-29]. For example, in response to DNA damage, p53, in most somatic cells, may induce cell cycle arrest at G1 through activation of its downstream target genes (e.g., p21), whereas, in ESCs, p53 cannot trigger the G1 checkpoint [24]. Qin et al. demonstrated that, in DNA-damaged hESCs, p53 cannot transactivate its downstream target Mdm2. The lack of Mdm2 induction disrupts the feedback degradation circuitry and promotes an overaccumulation of p53. The high level of p53 induces spontaneous apoptosis through the mitochondrial pathway to remove these abnormal stem cells, as well as spontaneous differentiation by suppressing pluripotency genes Oct- 4 and Nanog. When hESCs differentiate, the ability of p53 to activate the transcription of its target genes is restored [26]. In summary, apoptosis serves as a protective role in hESCs by eliminating stem cells that are abnormal and potentially dangerous from the pluripotent population [30].

hESCs, unlike mouse ESCs, are more vulnerable to apoptosis following cellular detachment and single-cell dissociation [31]. Such fragility upon dissociation has been a challenge for maintaining and developing hESC for biological research or for medical applications. After complete single-cell dissociation, hESCs undergo massive cell death, and the cloning efficiency of dissociated cells is generally less than $1 \%$ [32]. The apoptosis following cellular dissociation may be caused by disruption of the hESC microenvironment (i.e., niche), which is a consequence of alteration of signaling factors, cell-cell interactions (e.g., E-cadherin pathway), and cell-ECM interactions (e.g., integrin pathway). The niche is implicated in playing a pivotal role in hESC survival and self-renewal. Sasai's group demonstrated that, following dissociation, membrane expression of E-cadherin is irreparably disrupted, leading to activation of the ROCK (Rhoassociated kinase) pathway, particularly hyperactivation of actomyosin [32-34]. In addition, they showed that Y-27632 (a ROCK inhibitor) and Blebbistain (a myosin inhibitor) efficiently suppress dissociation-caused apoptosis. Moreover, Ding's group identified additional small molecules, Thiazovivin and Pyrintegrin markedly promote hESC survival after trypsin dissociation and enhance adhesion to matrigel- or laminin-coated plates (ECM-coated plates), but not gelatincoated plates. These compounds enhance cell-ECM adhesion and activate the integrin signaling pathway, which acts synergistically with growth factors to promote cell survival [34]. Collectively, these findings imply that ESCs maintain self-renewal through different functions of cell adhesion pathways and may explain the fundamental differences in morphology, growth factor requirement, and sensitivity to enzymatic cell dissociation.

In addition, maintenance of self-renewal capacity is critical for hESC preservation. The transcription factors, such as Oct4, Sox2, Nanog, and c-Myc, have essential roles in maintaining self-renewal. They form a regulatory circuitry of autoregulation and feedforward loops [35-37]. Interference with this regulatory circuitry may lead to apoptosis, differentiation, or cell senescence in ESC cultures. A recent study of hESCs demonstrated that sustained activation of the oncogene c-Myc induces apoptosis via activation of caspase and triggers differentiation by reduction of Oct 4 and Nanog expression [38]. 
In this section, we discussed the different apoptotic features of hESCs, with emphasis on the roles in genome integrity maintenance and the role in single-cell-dissociationinduced apoptosis (i.e., ROCK-dependent hESCs apoptosis). We hope these observations will improve culturing techniques for hESCs and lead to maintenance of self-renewal with minimal genetic instability, an essential requirement for the development of hESCs for human therapeutics.

\section{Apoptosis: Somatic Cell Reprogramming and the Role of p53}

Reprogramming of differentiated cells into the pluripotent, undifferentiated state is achieved by expression of three or four transcription factors, Oct4, Sox2, Klf4 (OSK), and cMyc (OSKM) [39]. The in vitro derived cells, called iPSCs, are dedifferentiated and resemble ESCs in many ways, including expression profiles and epigenetic status, albeit differences exist [40, 41]. Reprogramming towards pluripotency is a slow, stochastic process characterized by a large proportion of incompletely reprogrammed cells, and extensive cell death during the process of induction (Figure 1) [42]. Apoptosis occurs in the first few days following expression of the introduced reprogramming factors, and this phenomenon may contribute to the low efficiency of iPSC generation.

The critical role of apoptosis during somatic cell reprogramming is highlighted by the participation of p53 and the induction of apoptotic caspases by the 3- or 4-factor combination. As a crucial protein for tumor suppression, p53 plays different roles in senescence, apoptosis, cell cycle arrest, and DNA repair. Expression of c-Myc, one of the Yamanaka factors, shows the most dominant effect on induction of p53 and the downstream p21. However, omission of c-Myc and induction by OSK 3 -factor may also activate p53, suggesting a common transcriptional network leading to cellular stress activates the p53-dependent pathway.

Early experiments generating iPSC with 4-factors was inefficient $(\sim 0.05 \%)$, especially for human skin fibroblasts. Zhao et al. first demonstrated the role of p53 on the suppression of reprogramming efficiency [43]. RNAi knockdown of p53 improved the efficiency of iPSC generation by $\sim 100$ fold, even in the absence of c-Myc. Subsequently several important papers verified that p53 was a critical factor limiting iPSC induction [44-47]. Data from Belmonte's group demonstrated that knockdown of p53 or its target gene, p21 (also known as Cdkn1a), significantly increases reprogramming efficiency [45]. Based on the observation that the 3-factor cocktail induces the expression of the pro-apoptotic factor Bax (a p53-inducible gene), antagonizing reprogramming-induced apoptosis will also increase the number of iPSC colonies. Overexpression of $\mathrm{Bcl} 2$ (a Bax antagonist) suppresses apoptosis regardless of whether induction is initiated by two, three, or four reprogramming factors. Moreover, repression of $\mathrm{p} 19^{\text {Arf }}$ and $\mathrm{p} 16^{\text {Ink4a }}$ increases iPSC formation. p19 ${ }^{\text {Arf }}$ stabilizes p53 by inhibiting Mdm2, whereas $\mathrm{p} 16^{\text {Ink4a }}$ inhibits phosphorylation of the retinoblastoma gene product $(\mathrm{Rb})$ by cyclin $\mathrm{D}$-dependent kinases; reduction of both these factors rescues cell cycle arrest and improves reprogramming efficiency. Serrano's group found that the Ink4/Arf locus is epigenetically silenced in iPSC, but upregulated in aged cells, suggesting an important role of the Ink4a/Arf locus as an epigenetic barrier to reprogramming [48].

Similar experiments from Blasco's group support the role of p53 in activation of the DNA damage response and apoptosis in a p53-dependent mechanism. p53 is critically involved in the regulation of cell cycle arrest and apoptosis by preventing the propagation of DNA-damaged cells, including those with short or dysfunctional telomeres. Using genetically modified MEF cells that lack telomerase $\left(\mathrm{Terc}^{-/-}\right.$), they showed a significant proportion (10\% and $15 \%)$ of wildtype cells undergo apoptosis, whereas it is further increased to $40 \%$ in G3 Terc $^{-/-}$cells at days 9-13 after infection. Knockdown of p53 or overexpression of Bcl2 abrogates apoptosis at the onset of iPSC induction, allowing cells with preexisting damaged or dysfunctional telomeres to reprogram [46]. These studies indicate that p53 limits reprogramming by inducing apoptosis at the time of pluripotency induction. Consistent with other investigators, Yamanaka's group showed the involvement of the p53-p21 pathway as a barrier to iPSC generation. In the p53-null background, induction efficiency is boosted to $10 \%$. Even terminally differentiated T lymphocytes can be successfully reprogrammed [44].

Li et al. documented the important finding of caspase involvement during reprogramming [49]. Two key apoptotic proteases, caspases 3 and 8, are activated following transduction of the reprogramming factors (c-Myc was excluded in their experiments because it is able to activate caspases and induce apoptosis). Oct4, the indispensable factor for iPSC generation, is responsible for activation of caspases 3 and 8. In agreement with Blasco's group, they found $10-20 \%$ of cells undergo apoptosis following OSKM factor induction. However, in the absence of c-Myc using the OSNL factors, apoptosis is reduced to $1-3 \%$, suggesting a critical role of c-Myc in the activation of apoptosis. The activation of caspases during reprogramming eliminates cells by apoptosis, meanwhile benefits iPSC induction, as shown by a significant reduction of iPSC formation after knockdown of caspases 3 or 8 . The importance of caspase activation in promoting reprogramming is demonstrated by its capacity to degrade $\mathrm{Rb}$, which is a tumor suppressor gene that regulates cell cycle progression and helps the promotion and maintenance of cellular differentiation. It appears that the $\mathrm{Rb}$ and p53 pathways work independently to suppress iPSC formation; however, the exact mechanism remains to be uncovered.

Reprogramming involves extensive chromatin remodeling and induction of the pluripotency network to reset the epigenome to an ESC-like state [50]. During the process reactive oxygen species (ROS) are generated by metabolism [51]. Increased levels of ROS leads to DNA damage, cell senescence, and apoptosis. ROS may hinder survival of reprogramming cells, as suggested by observations of enhancement of iPSC generation during hypoxia $[47,52]$. In addition, mitochondrial content and oxidative stress are repressed in iPSCs or hESCs [53], suggesting that ROS generation by reprogramming factors is unfavorable for generating iPSCs. Esteban et al. identified vitamin $\mathrm{C}$ as an effective 


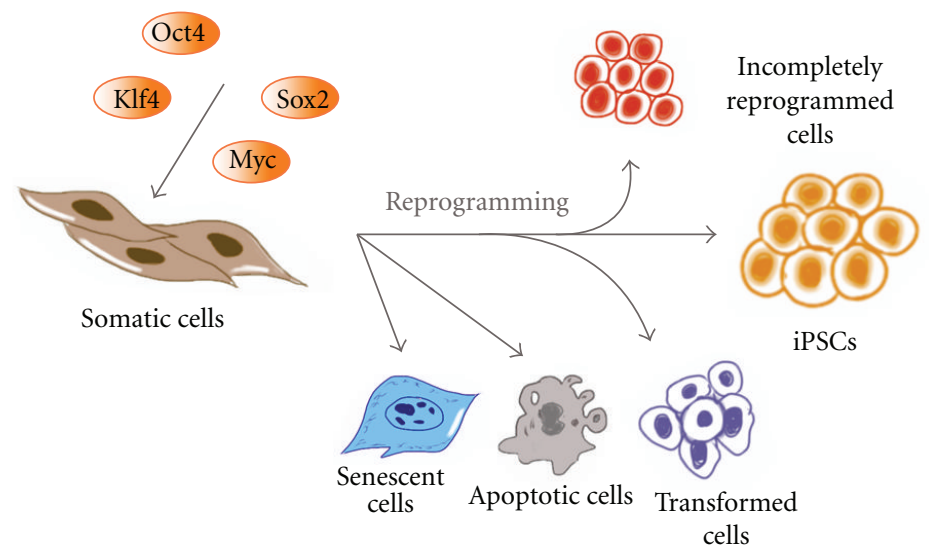

FIGURE 1: Different cell fates arisen from introduction of reprogramming factors. Overexpression of Oct4, Sox2, Klf4, and c-Myc results in generation of apoptotic cells, senescent cells, transformed cells, incompletely reprogrammed cells, and ESC-like iPSCs. During the course of induction of pluripotency, many cells undergo apoptosis or become senescent. A large proportion of cells remained in culture represent incompletely reprogrammed or become transformed.

chemical to boost iPSC generation; however surprisingly the underlying mechanism seems not totally dependent on its antioxidant activity [51]. Instead, treatment of vitamin $\mathrm{C}$ reduces p53/p21 levels, which is the main barrier to successful reprogramming. They further identified histone demethylases Jhdm $1 \mathrm{a} / 1 \mathrm{~b}$ as the downstream effectors of vitamin C [54]. In particular, Jhdm1b promotes cell cycle progression and suppresses senescence by repressing the Ink/Arf locus during reprogramming. Furthermore, inhibition of the mTOR pathway by rapamycin or PP242 or insulin/IGF-1 signaling pathway notably enhances the efficiency of reprogramming [55]. From the concept that reprogramming is a stressful process that activates apoptosis and cellular senescence, targeting the mTOR pathway alleviates senescence imposed by DNA damage response [56]. These findings support the role of senescence as a limiting factor for successful reprogramming.

In summary, introduction of reprogramming factors appears to exert a cellular stress that will lead to DNA damage, senescence, and apoptosis. Activation of p53 and other apoptotic players is a protective mechanism that eliminates damaged or senescent cells from continuing replication, a similar mechanism that p53 does by suppressing tumorigenesis (Figure 2).

\section{Cellular Senescence, Accelerated Aging, and Reprogramming}

Transcription-factor-based reprogramming results in generation of different fates of somatic cells. The reprogramming factors can trigger cellular senescence and apoptosis, leading to cell death [53]. For those cells that can bypass the stress, the majority form transformed cells, partially reprogrammed
iPSCs, and very low percentage of cells form true iPSCs. The Ink4a/Arf locus is epigenetically silenced in iPSC or ESC, but expressed in differentiated fibroblasts [46]. Ink4a/Arf expression is associated with natural aging and with passage number in culture $[46,47]$. Activation of the Ink4a/Arf locus is found during reprogramming and becomes a ratelimitation in iPSC generation. This observation is consistent with the upregulation of the $\mathrm{p} 16^{\text {Ink4a }}$ and $\mathrm{p} 19^{\text {Arf }}$ in aged fibroblasts, which display a lower reprogramming efficiency. $\mathrm{p} 19^{\mathrm{Arf}}$ and $\mathrm{p} 16^{\text {Ink4a }}$ regulate $\mathrm{p} 53$ and $\mathrm{Rb}$ tumor suppressors respectively, both of them are important players in cell cycle progression and proliferation.

Reprogramming of aged or senescent cells becomes technically challenging [57], given the fact that these cells show slow rates of proliferation, shortening of the telomere, increased DNA damage, and upregulation of p53 and Ink4a/Arf genes. MEF cells from the third-generation mice deficient in telomerase carry accumulated short telomeres and can barely be reprogrammed [58]. However, this defect can be ameliorated by reintroduction of telomerase or suppression of p53 $[46,58]$. Remarkably, in p53 ${ }^{-/-}$background, iPSC derived from telomerase-deficient MEF cells show even further shortening of telomeres, suggesting that inhibition of p 53 technically bypasses the p53-dependent cell death, but does not "rejuvenate" the reprogrammed iPSCs with elongated telomeres [46]. In humans, the shortened telomere is generally correlated with aging. Despite that, human iPSCs have been successfully derived from skin biopsies of old patients of 82 and 89 years old, although the efficiency of iPSC generation was not comparable to young donors [59]. Recently, senescent fibroblasts derived by prolonged culture (51 PDs) or fibroblasts directly taken from centenarians were reprogrammed with a six-factor cocktail 


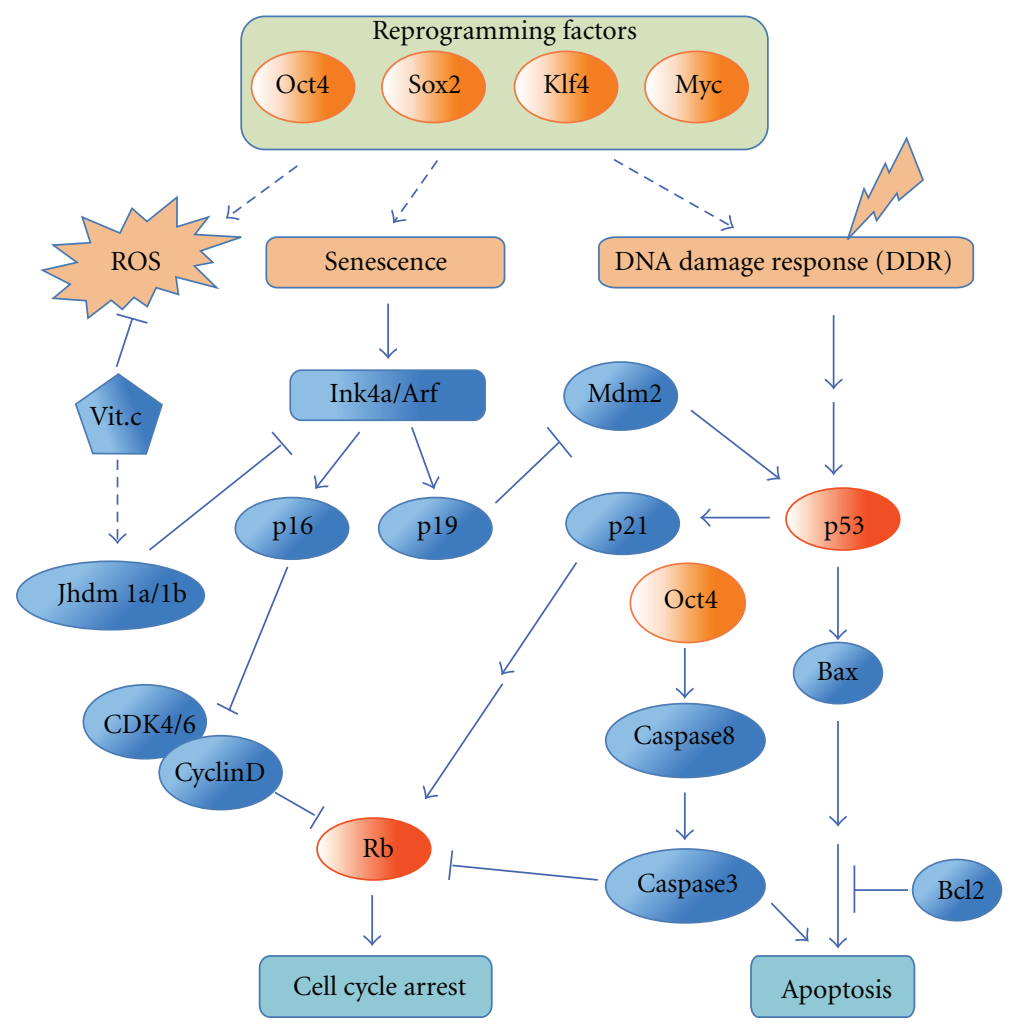

FIGURE 2: The critical role of p53 tumor suppressors in limiting the generation of iPSC by induction of apoptosis or cell cycle arrest. Cell reprogramming is a stressful process accompanied with generation of ROS, induction of senescence, and DNA damage response. Activation of p53 and its downstream p21 observed during reprogramming is likely induced by DNA damage response. p21 is a cyclin-dependent kinase (CDK) inhibitor that regulates cell cycle arrest. Besides, senescence-induced expressions of p16 and p19 indirectly activate tumor suppressors p53 and $\mathrm{Rb}$, respectively, leading to cell cycle arrest and apoptosis. Oct4-dependent activation of caspase 8 and 3 has a dual role in promoting apoptosis and cleavage of $\mathrm{Rb}$, which favors reprogramming. Treatment of cells with Vitamin $\mathrm{C}$ also facilitates iPSC formation by scavenging ROS and meanwhile regulates histone demethylases Jhdmla/lb, the latter of which is able to promote cell cycle progression and suppress senescence by repressing the Ink4/Arf locus. Double arrows: multiple steps.

(OSKMNL). The derived iPSCs reset telomere length and are indistinguishable from normal hESCs [60]. Interestingly, additional reprogramming factors NANOG and LIN28 are essential for generating iPSC for these senescent cells, and a longer time of induction is required, implicating certain intrinsic senescence barriers that need to be overcome by these additional factors.

Reprogramming differentiated cells to iPSC is analogies to rejuvenation of "aged" cells to their ground state, allowing these pluripotent cells to redifferentiate into different lineages. An alternative excellent model for the study of senescence and reprogramming potentially is the premature aging syndromes derived from patients who display accelerated aging phenotypes such as premature grey hair (or alopecia), arteriosclerosis, heart disease, and shortened lifespan. The Hutchinson-Gilford progeria syndrome (HGPS) is a segmental progeroid syndrome that occurs in children with dominant mutation of the LMNA gene. The aberrantly spliced form of the LMNA gene product (progerin) causes irregular nuclear organization and loss of functional integrity of the nuclear lamina. Accumulation of DNA damage (doublestrand breaks and abnormal DNA damage response) and cell cycle arrest are commonly found in HGPS cells [61]. Secondly, progerin induces premature aging by increasing DNA damage signaling and activation of $\mathrm{p} 53$ and Rb pathways. Progerin-induced DNA damage signaling is localized to telomeres and induces dysfunction of telomeres, indicative of an accelerated aging phenotype in HGPS cells [62]. Reprogramming of HGPS skin fibroblasts to iPSC has been reported by different groups [63-65]. The derived HGPSiPSCs demonstrate absence of progerin expression, normal nuclear structure, and epigenetic markers. However upon differentiation, abnormal cellular phenotypes associated with this syndrome reappear. Zhang et al. further demonstrated that DNA damage and nuclear abnormalities recur in redifferentiated mesenchymal stem cells (MSCs), vascular smooth muscle cells, and fibroblasts, recapitulating the aging phenotype of HGPS [63]. Questions still remain whether these reprogrammed patient iPSCs can properly reset the dysfunctional telomeres to the normal state. Another progeroid syndrome, the Werner syndrome (WS), is an accelerated aging phenotype of adults caused by recessive mutation of the DNA helicase WRN [66]. No iPSCs have been derived from WS patients to date. The lessons we learned 
about the mechanisms of cellular senescence and reprogramming involving central safe guardians such as $\mathrm{p} 53 / \mathrm{Rb}$ and the barrier of senescence like Ink4/Arf and telomere shortening may provide insights into technical refinement for derivation of iPSC from senescent cells and as a tool for modeling agingrelated diseases.

\section{Concluding Remarks and Future Perspectives}

In this paper we discussed the multiple roles of apoptosis in ESC and iPSC induction. We highlighted the importance of apoptotic caspases and p53 in the activation of apoptosis and cellular senescence. The knowledge we learn from these recent studies uncovers a previously unknown function of p53 in safeguarding reprogramming in addition to its wellknown function in tumor suppression. Here we summarize two important points.

First, understanding the role of apoptosis in ESC and somatic cell reprogramming to iPSC may help us evaluate the current technology aiming at making clinically "safe" iPSC for cell therapy in the future. A major concern of iPSCbased therapy is the tumorigenicity of iPSC, particularly for the risk of overexpressing Klf4 and c-Myc, both of them are known oncogenes [67]. The latter has been found to induce apoptosis and differentiation in human ESC and trigger cellular senescence during reprogramming [38]. Additionally, the pivotal role of p53 activation during reprogramming appears to be a self-defense mechanism that prevents cells from oncogenic transformation. Whereas p53 mutation is commonly found in many cancers, the inhibition of p53 for boosting iPSC generation is highly risky for generating clinical grade iPSC, as demonstrated by Marión et al. that preexisting DNA damage and dysfunctional telomeres are still present in p53-null iPSC [46]. The similarity between oncogenesis and reprogramming raises concerns about the utilization of these p53-null iPSCs for therapy. Non-transcriptionfactor-based reprogramming methods by using microRNAs and small molecules as examples are potentially good alternatives, although the safety of these iPSCs needs to be assessed [68]. The future direction is to develop safe protocols for generating clinical grade iPSCs with high efficiency and low risk of tumorigenesis without the need of any genetic modification.

Second, understanding the role of senescence in reprogramming provides a molecular basis for deriving iPSC from aging-related diseases such as HGPS and WS. For instance, to reprogram premature aged somatic cells from WS, inhibition of $\mathrm{p} 53 / \mathrm{p} 21$ or Ink4a/Arf may help to overcome the preexisting senescent barrier and dysfunctional telomeres found in these cells. It is also interesting to understand whether somatic cell reprogramming can properly reset the accumulative epigenetic changes arising from natural aging or premature aging to a normal state. We foresee that reversing senescence in aged cells will have clinical implications for developing cell-based therapy for the treatment of aged individuals and patients of progeria.

\section{Acknowledgment}

This work was supported by Division of Intramural Research of The Eunice Kennedy Shriver National Institute of Child Health and Human Development, National Institutes of Health.

\section{References}

[1] S. Elmore, "Apoptosis: a review of programmed cell death," Toxicologic Pathology, vol. 35, no. 4, pp. 495-516, 2007.

[2] A. D. Schimmer, "Apoptosis in leukemia: from molecular pathways to targeted therapies," Best Practice and Research: Clinical Haematology, vol. 21, no. 1, pp. 5-11, 2008.

[3] R.S.Y. Wong, "Apoptosis in cancer: from pathogenesis to treatment," Journal of Experimental and Clinical Cancer Research, vol. 30, no. 1, 2011.

[4] A. Russo, M. Terrasi, V. Agnese, D. Santini, and V. Bazan, "Apoptosis: a relevant tool for anticancer therapy," Annals of Oncology, vol. 17, supplement 7, pp. vii115-vii123, 2006.

[5] I. H. Song, Y. S. Kim, and T. H. Kim, "Role of apoptotic and necrotic cell death under physiologic conditions," Journal of Biochemistry and Molecular Biology, vol. 41, no. 1, pp. 1-10, 2008.

[6] J. E. Belizário, I. Alves, J. M. Occhiucci, M. Garay-Malpartida, and A. Sesso, "A mechanistic view of mitochondrial death decision pores," Brazilian Journal of Medical and Biological Research, vol. 40, no. 8, pp. 1011-1024, 2007.

[7] Y. Fuchs and H. Steller, "Programmed cell death in animal development and disease," Cell, vol. 147, no. 4, pp. 742-758, 2011.

[8] N. Edison, D. Zuri, I. Maniv et al., "The IAP-antagonist ARTS initiates caspase activation upstream of cytochrome C and SMACDiablo," Cell Death and Differentiation, vol. 19, no. 2, pp. 356-368, 2012.

[9] K. H. Vousden and C. Prives, "Blinded by the light: the growing complexity of p53," Cell, vol. 137, no. 3, pp. 413-431, 2009.

[10] D. P. Lane, “Cancer. p53, guardian of the genome," Nature, vol. 358, no. 6381, pp. 15-16, 1992.

[11] V. Zuckerman, K. Wolyniec, R. V. Sionov, S. Haupt, and Y. Haupt, "Tumour suppression by p53: the importance of apoptosis and cellular senescence," Journal of Pathology, vol. 219, no. 1, pp. 3-15, 2009.

[12] L. Yang, D. Wu, K. Luo, S. Wu, and P. Wu, "Andrographolide enhances 5-fluorouracil-induced apoptosis via caspase-8dependent mitochondrial pathway involving p53 participation in hepatocellular carcinoma (SMMC-7721) cells," Cancer Letters, vol. 276, no. 2, pp. 180-188, 2009.

[13] J. E. Chipuk, T. Kuwana, L. Bouchier-Hayes et al., "Direct activation of bax by p53 mediates mitochondrial membrane permeabilization and apoptosis," Science, vol. 303, no. 5660, pp. 1010-1014, 2004.

[14] M. P. Chao, R. Majeti, and I. L. Weissman, "Programmed cell removal: a new obstacle in the road to developing cancer," Nature Reviews Cancer, vol. 12, no. 1, pp. 58-67, 2012.

[15] D. V. Vujaklija, S. Sucic, T. Gulic, M. Dominovic, and D. Rukavina, "Cell death mechanisms at the maternal-fetal interface: insights into the role of granulysin," Clinical and Developmental Immunology, vol. 2012, Article ID 180272, 8 pages, 2012.

[16] I. Boumela, S. Assou, A. Aouacheria et al., "Involvement of BCL2 family members in the regulation of human oocyte and 
early embryo survival and death: gene expression and beyond," Reproduction, vol. 141, no. 5, pp. 549-561, 2011.

[17] J. Ramalho-Santos, S. Varum, S. Amaral, P. C. Mota, A. P. Sousa, and A. Amaral, "Mitochondrial functionality in reproduction: from gonads and gametes to embryos and embryonic stem cells," Human Reproduction Update, vol. 15, no. 5, pp. 553-572, 2009.

[18] K. Takahashi and S. Yamanaka, "Induction of pluripotent stem cells from mouse embryonic and adult fibroblast cultures by defined factors," Cell, vol. 126, no. 4, pp. 663-676, 2006.

[19] A. Rufini and G. Melino, "Cell death pathology: the war against cancer," Biochemical and Biophysical Research Communications, vol. 414, no. 3, pp. 445-450, 2011.

[20] J. A. Thomson, "Embryonic stem cell lines derived from human blastocysts," Science, vol. 282, no. 5391, pp. 1145-1147, 1998.

[21] J. Yu and J. A. Thomson, "Pluripotent stem cell lines," Genes and Development, vol. 22, no. 15, pp. 1987-1997, 2008.

[22] C. E. Murry and G. Keller, "Differentiation of embryonic stem cells to clinically relevant populations: lessons from embryonic development," Cell, vol. 132, no. 4, pp. 661-680, 2008.

[23] P. J. Stambrook and E. D. Tichy, "Preservation of genomic integrity in mouse embryonic stem cells," Advances in Experimental Medicine and Biology, vol. 695, pp. 59-75, 2010.

[24] E. D. Tichy, "Mechanisms maintaining genomic integrity in embryonic stem cells and induced pluripotent stem cells," Experimental Biology and Medicine, vol. 236, no. 9, pp. 987-996, 2011.

[25] K. H. Lee, M. Li, A. M. Michalowski et al., "A genomewide study identifies the Wnt signaling pathway as a major target of p53 in murine embryonic stem cells," Proceedings of the National Academy of Sciences of the United States of America, vol. 107, no. 1, pp. 69-74, 2010.

[26] H. Qin, T. Yu, T. Qing et al., "Regulation of apoptosis and differentiation by 553 in human embryonic stem cells," Journal of Biological Chemistry, vol. 282, no. 8, pp. 5842-5852, 2007.

[27] V. Solozobova and C. Blattner, "Regulation of p53 in embryonic stem cells," Experimental Cell Research, vol. 316, no. 15, pp. 2434-2446, 2010.

[28] B. T. Spike and G. M. Wahl, "P53, stem cells, and reprogramming: tumor suppression beyond guarding the genome," Genes and Cancer, vol. 2, no. 4, pp. 404-419, 2011.

[29] T. Zhao and Y. Xu, "p53 and stem cells: new developments and new concerns," Trends in Cell Biology, vol. 20, no. 3, pp. 170$175,2010$.

[30] R. Sarig and V. Rotter, "Can an iPS cell secure its genomic fidelity?" Cell Death \& Differentiation, vol. 18, no. 5, pp. 743744, 2011.

[31] M. Ohgushi and Y. Sasai, "Lonely death dance of human pluripotent stem cells: ROCKing between metastable cell states," Trends in Cell Biology, vol. 21, no. 5, pp. 274-282, 2011.

[32] K. Watanabe, M. Ueno, D. Kamiya et al., "A ROCK inhibitor permits survival of dissociated human embryonic stem cells," Nature Biotechnology, vol. 25, no. 6, pp. 681-686, 2007.

[33] M. Ohgushi, M. Matsumura, M. Eiraku et al., "Molecular pathway and cell state responsible for dissociation-induced apoptosis in human pluripotent stem cells," Cell Stem Cell, vol. 7, no. 2, pp. 225-239, 2010.

[34] Y. Xu, X. Zhu, H. S. Hahm et al., "Revealing a core signaling regulatory mechanism for pluripotent stem cell survival and self-renewal by small molecules," Proceedings of the National Academy of Sciences of the United States of America, vol. 107, no. 18 , pp. 8129-8134, 2010.
[35] L. A. Boyer, I. L. Tong, M. F. Cole et al., "Core transcriptional regulatory circuitry in human embryonic stem cells," Cell, vol. 122, no. 6, pp. 947-956, 2005.

[36] N. Liu, M. Lu, X. Tian, and Z. Han, "Molecular mechanisms involved in self-renewal and pluripotency of embryonic stem cells," Journal of Cellular Physiology, vol. 211, no. 2, pp. 279286, 2007.

[37] R. Stewart, M. Stojkovic, and M. Lako, "Mechanisms of selfrenewal in human embryonic stem cells," European Journal of Cancer, vol. 42, no. 9, pp. 1257-1272, 2006.

[38] T. Sumi, N. Tsuneyoshi, N. Nakatsuji, and H. Suemori, "Apoptosis and differentiation of human embryonic stem cells induced by sustained activation of c-Myc," Oncogene, vol. 26, no. 38, pp. 5564-5576, 2007.

[39] K. Takahashi and S. Yamanaka, "Induction of pluripotent stem cells from mouse embryonic and adult fibroblast cultures by defined factors," Cell, vol. 126, no. 4, pp. 663-676, 2006.

[40] Y. Mayshar, U. Ben-David, N. Lavon et al., "Identification and classification of chromosomal aberrations in human induced pluripotent stem cells," Cell Stem Cell, vol. 7, no. 4, pp. 521$531,2010$.

[41] A. Gore, Z. Li, H. -L. Fung et al., "Somatic coding mutations in human induced pluripotent stem cells," Nature, vol. 471, no. 7336, pp. 63-67, 2011.

[42] J. Hanna, K. Saha, B. Pando et al., "Direct cell reprogramming is a stochastic process amenable to acceleration," Nature, vol. 462, no. 7273, pp. 595-601, 2009.

[43] Y. Zhao, X. Yin, H. Qin et al., "Two supporting factors greatly improve the efficiency of human IPSC generation," Cell Stem Cell, vol. 3, no. 5, pp. 475-479, 2008.

[44] H. Hong, K. Takahashi, T. Ichisaka et al., "Suppression of induced pluripotent stem cell generation by the p53-p21 pathway," Nature, vol. 460, no. 7259, pp. 1132-1135, 2009.

[45] T. Kawamura, J. Suzuki, Y. V. Wang et al., "Linking the p53 tumour suppressor pathway to somatic cell reprogramming," Nature, vol. 460, no. 7259, pp. 1140-1144, 2009.

[46] R. M. Marión, K. Strati, H. Li et al., "A p53-mediated DNA damage response limits reprogramming to ensure iPS cell genomic integrity," Nature, vol. 460, no. 7259, pp. 1149-1153, 2009.

[47] J. Utikal, J. M. Polo, M. Stadtfeld et al., "Immortalization eliminates a roadblock during cellular reprogramming into iPS cells," Nature, vol. 460, no. 7259, pp. 1145-1148, 2009.

[48] H. Li, M. Collado, A. Villasante et al., "The Ink4/Arf locus is a barrier for iPS cell reprogramming," Nature, vol. 460, no. 7259, pp. 1136-1139, 2009.

[49] F. Li, Z. He, J. Shen et al., "Apoptotic caspases regulate induction of iPSCs from human fibroblasts," Cell Stem Cell, vol. 7, no. 4, pp. 508-520, 2010.

[50] K. Plath and W. E. Lowry, "Progress in understanding reprogramming to the induced pluripotent state," Nature Reviews Genetics, vol. 12, no. 4, pp. 253-265, 2011.

[51] M. A. Esteban, T. Wang, B. Qin et al., "Vitamin C enhances the generation of mouse and human induced pluripotent stem cells," Cell Stem Cell, vol. 6, no. 1, pp. 71-79, 2010.

[52] Y. Yoshida, K. Takahashi, K. Okita, T. Ichisaka, and S. Yamanaka, "Hypoxia enhances the generation of induced pluripotent stem cells," Cell Stem Cell, vol. 5, no. 3, pp. 237241, 2009.

[53] N. Mah, Y. Wang, M. -C. Liao et al., "Molecular insights into reprogramming-initiation events mediated by the OSKM gene regulatory network," PLoS One, vol. 6, no. 8, article e24351, 2011. 
[54] T. Wang, K. Chen, X. Zeng et al., "The histone demethylases Jhdm $1 \mathrm{a} / 1 \mathrm{~b}$ enhance somatic cell reprogramming in a vitaminC-dependent manner," Cell Stem Cell, vol. 9, no. 6, pp. 575587, 2011.

[55] T. Chen, L. Shen, J. Yu et al., "Rapamycin and other longevitypromoting compounds enhance the generation of mouse induced pluripotent stem cells," Aging Cell, vol. 10, no. 5, pp. 908-911, 2011.

[56] J. A. Menendez, L. Vellon, C. Oliveras-Ferraros, S. Cufí, and A. Vazquez-Martin, "mTOR-regulated senescence and autophagy during reprogramming of somatic cells to pluripotency: a roadmap from energy metabolism to stem cell renewal and aging," Cell Cycle, vol. 10, no. 21, pp. 3658-3677, 2011.

[57] A. Banito, S. T. Rashid, J. C. Acosta et al., "Senescence impairs successful reprogramming to pluripotent stem cells," Genes and Development, vol. 23, no. 18, pp. 2134-2139, 2009.

[58] R. M. Marion, K. Strati, H. Li et al., "Telomeres Acquire Embryonic Stem Cell Characteristics in Induced Pluripotent Stem Cells," Cell Stem Cell, vol. 4, no. 2, pp. 141-154, 2009.

[59] J. T. Dimos, K. T. Rodolfa, K. K. Niakan et al., "Induced pluripotent stem cells generated from patients with ALS can be differentiated into motor neurons," Science, vol. 321, no. 5893, pp. 1218-1221, 2008.

[60] L. Lapasset, O. Milhavet, A. Prieur et al., "Rejuvenating senescent and centenarian human cells by reprogramming through the pluripotent state," Genes and Development, vol. 25, no. 21, pp. 2248-2253, 2011.

[61] P. R. Musich and Y. Zou, "DNA-damage accumulation and replicative arrest in Hutchinson-Gilford progeria syndrome," Biochemical Society Transactions, vol. 39, no. 6, pp. 1764-1769, 2011.

[62] E. K. Benson, S. W. Lee, and S. A. Aaronson, "Role of progerininduced telomere dysfunction in HGPS premature cellular senescence," Journal of Cell Science, vol. 123, no. 15, pp. 26052612, 2010.

[63] J. Zhang, Q. Lian, G. Zhu et al., "A Human iPSC model of hutchinson gilford progeria reveals vascular smooth muscle and mesenchymal stem cell defects," Cell Stem Cell, vol. 8, no. 1, pp. 31-45, 2010.

[64] G. H. Liu, B. Z. Barkho, S. Ruiz et al., "Recapitulation of premature ageing with iPSCs from Hutchinson-Gilford progeria syndrome," Nature, vol. 472, no. 7342, pp. 221-227, 2011.

[65] J. C. Ho, T. Zhou, W. H. Lai et al., "Generation of induced pluripotent stem cell lines from 3 distinct laminopathies bearing heterogeneous mutations in lamin A/C," Aging, vol. 3, no. 4, pp. 380-390, 2011.

[66] L. Domínguez-Gerpe and D. Araújo-Vilar, "Prematurely aged children: molecular alterations leading to Hutchinson-Gilford progeria and Werner syndromes," Current aging science, vol. 1, no. 3, pp. 202-212, 2008.

[67] U. Ben-David and N. Benvenisty, "The tumorigenicity of human embryonic and induced pluripotent stem cells," Nature Reviews Cancer, vol. 11, no. 4, pp. 268-277, 2011.

[68] F. Anokye-Danso, C. M. Trivedi, D. Juhr et al., "Highly efficient miRNA-mediated reprogramming of mouse and human somatic cells to pluripotency," Cell Stem Cell, vol. 8, no. 4, pp. 376-388, 2011. 

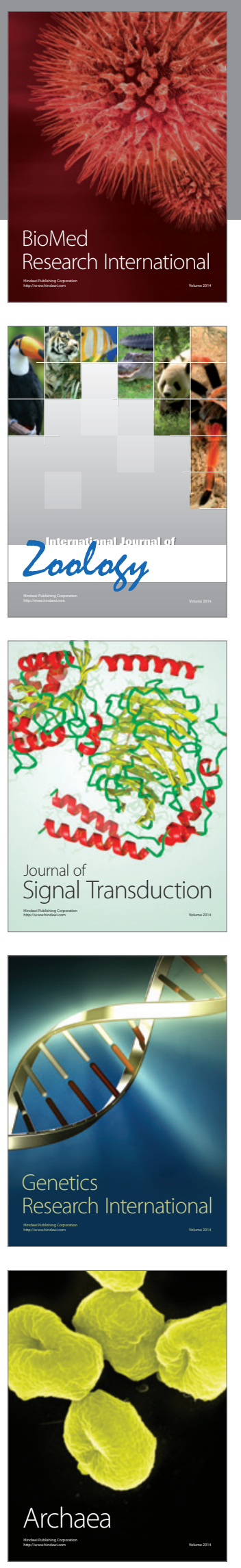
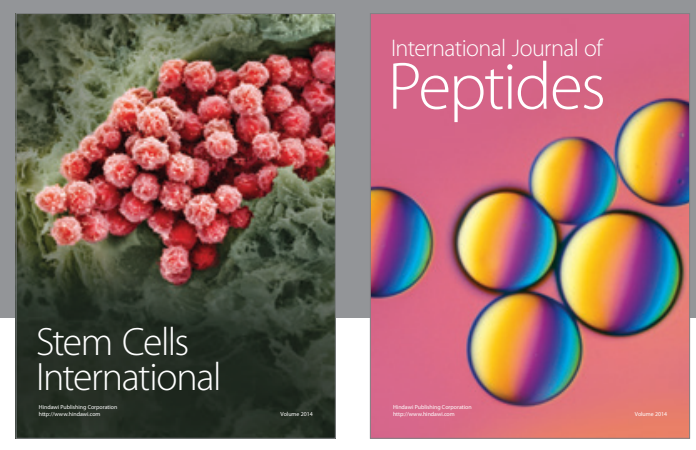

Submit your manuscripts at

http://www.hindawi.com
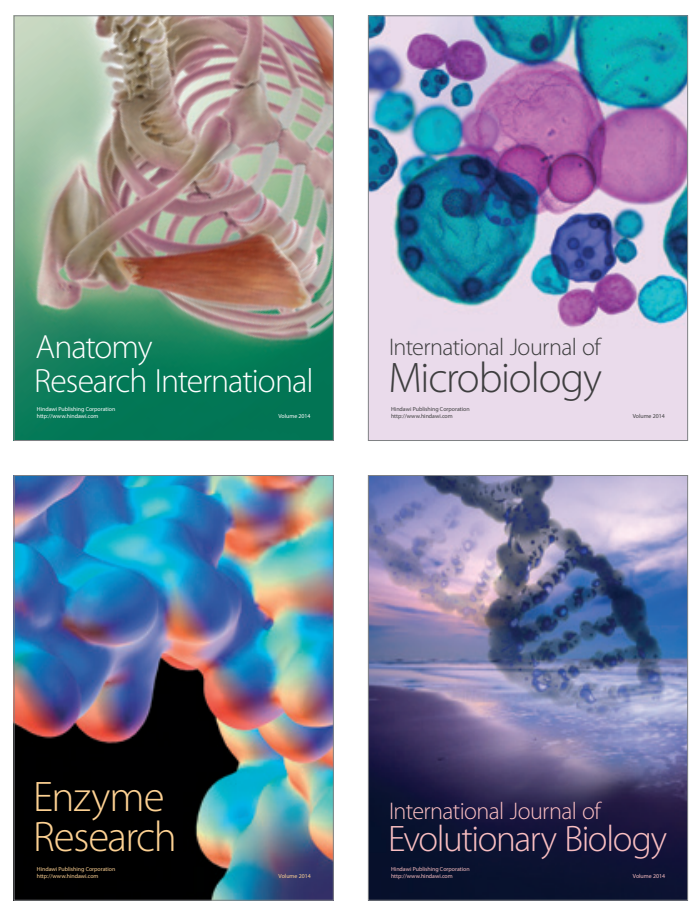
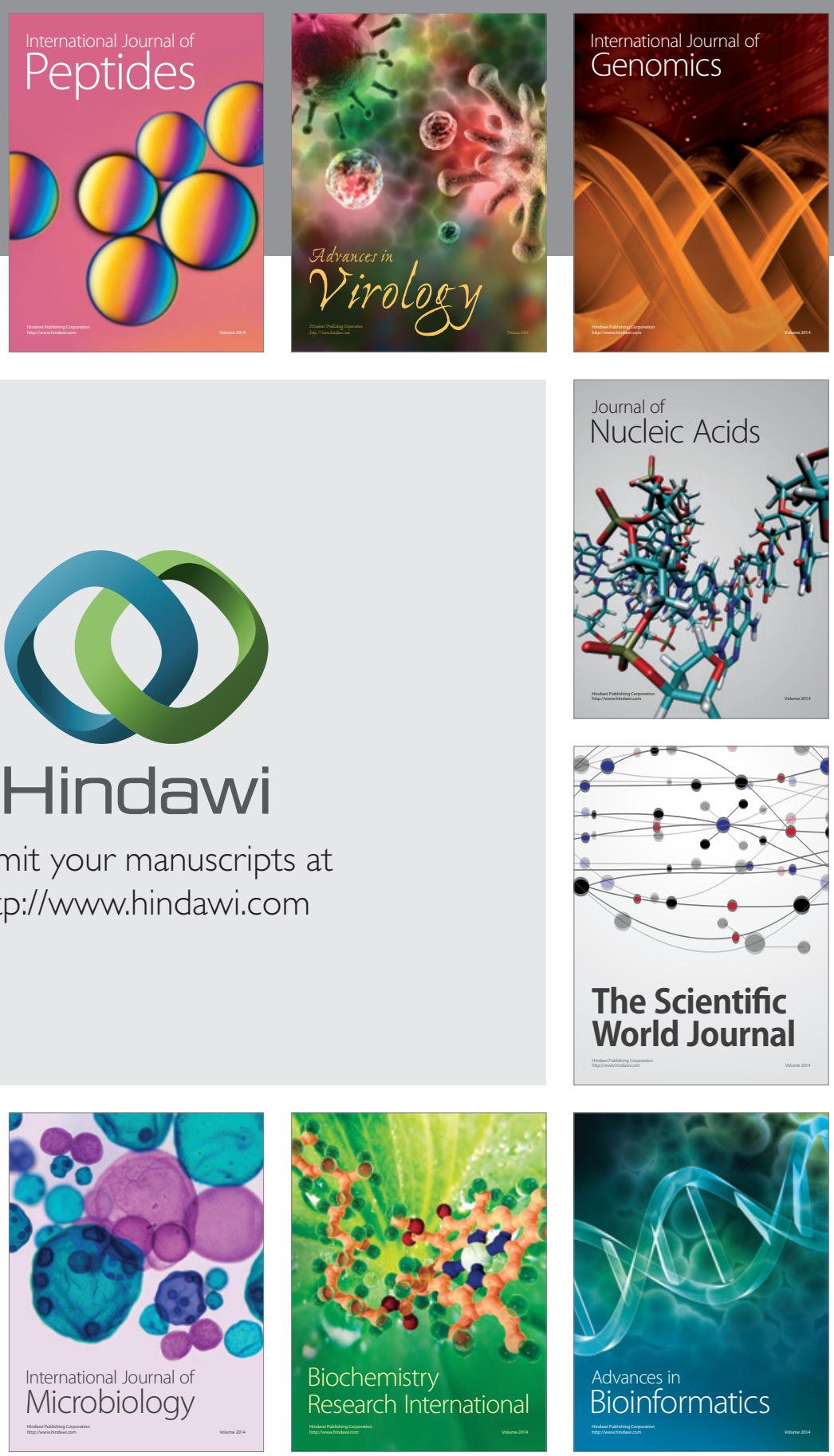

The Scientific World Journal
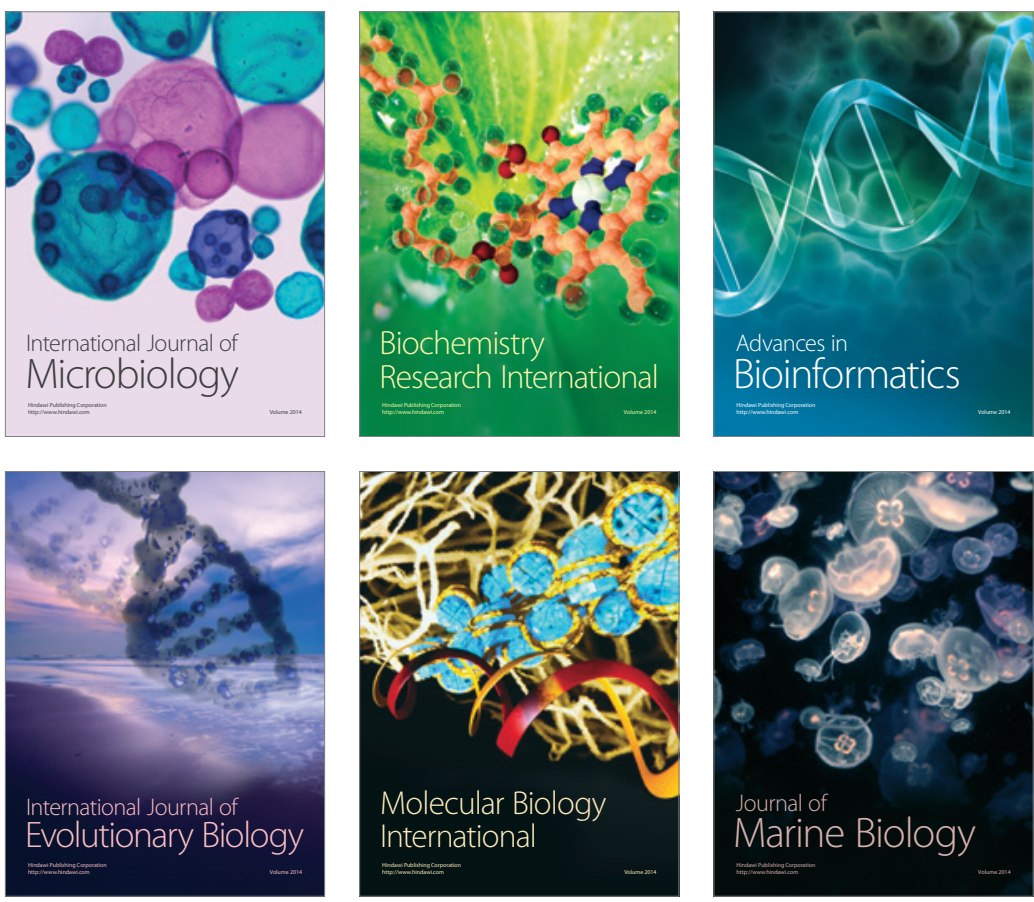